Journal of Quantitative Spectroscopy \& Radiative Transfer 81 (2003) 385-394
Journal of Quantitative

Spectroscopy \& Radiative

Transfer

www.elsevier.com/locate/jqsrt

\title{
Spectroscopic characterization of plasma densities of laser-irradiated Al foils
}

\author{
O. Renner ${ }^{\mathrm{a}, \mathrm{c}, *}$, J. Limpouch ${ }^{\mathrm{b}}$, E. Krousky ${ }^{\mathrm{a}}$, I. Uschmann ${ }^{\mathrm{c}}$, E. Förster ${ }^{\mathrm{c}}$ \\ ${ }^{a}$ Institute of Physics, Academy of Sciences CR, Na Slovance 2, CZ-18221 Prague, Czech Republic \\ ${ }^{\mathrm{b}}$ Czech Technical University, Faculty of Nuclear Sciences and Physical Engineering, Brehova 7, \\ CZ-11519 Prague, Czech Republic \\ ${ }^{\mathrm{c}}$ Institut fur Optik und Quantenelektronik, Friedrich-Schiller-Universitat, Max-Wien-Platz 1, \\ D-07743 Jena, Germany
}

Accepted 15 February 2003

\begin{abstract}
Detailed spectroscopic analysis of K-shell emission simultaneously observed at the irradiated and unirradiated surface of laser-exploded foils is reported. The space-dependent spectral line widths and relative shifts in the positions of the Al Lyman transition are interpreted in terms of the plasma density variations; the trend in the shifts data supports the idea of a density-dependent line shift. The significant features of the spectra emitted from the densest part of the plasma are compared with predictions from standard diagnostics modeling based on codes MEDUSA and FLY, and with the results of 2D ATLANT hydrodynamic simulations post-processed with a novel suite of atomic physics codes XEPAP. The results obtained corroborate the feasibility of the plasma density diagnostics based on line shifts in the intermediately coupled plasmas.
\end{abstract}

(C) 2003 Elsevier Science Ltd. All rights reserved.

Keywords: X-ray spectra; Spectral line shapes; Density diagnostics; Plasma simulation

\section{Introduction}

X-ray spectra emitted from hot dense plasmas provide an important diagnostic tool to infer local plasma parameters particularly ionization stage, density, and temperature [1]. The spectroscopic measurements of the plasma density are typically based on absolute continuum or line intensities, relative line intensities or spectral line widths and profiles. Most of these techniques require some

\footnotetext{
${ }^{*}$ Corresponding author. Institut fur Optik und Quantenelktronik, Friedrich Schiller Universitat, Max Wien Platz 1, Jena 07443, Germany. Tel.: +49-3641-947263; fax: +49-3641-947262.

E-mail address: renner@ioq.uni-jena.de (O. Renner).
} 
knowledge of plasma temperature, which must be provided either by independent measurements, e.g., by Langmuir probe or Thomson scattering diagnostics, or by spectroscopic methods [2].

According to theoretical predictions [3-6], the widths and frequency shifts of Stark-broadened lines are strongly density dependent and relatively weakly temperature dependent. Consequently, the line widths of hydrogenic ions are standardly used for density diagnosis of laboratory plasmas. Concerning the line shifts, the situation is less clear as efforts that aimed to develop new robust high-density diagnostics failed [7]. Red line shifts in dense laser-produced plasmas observed in a number of earlier experiments are briefly reviewed, e.g., in Refs. [8,9]. These measurements were mostly directed to provide a benchmark for competing theories of line shifts and to investigate the physics of ions in high-density plasmas. Recently, the interest in the plasma-induced line shifts reappeared with respect to directly or indirectly driven microsphere experiments $[9,10]$ characterized by high electron densities and temperatures, i.e., $10^{24} \mathrm{~cm}^{-3}$ and $1 \mathrm{keV}$, respectively, and distinct line shifts. Further, the analyses of the extremely broad Al K-shell resonance lines and their satellites observed in short pulse laser-produced plasma experiments at solid density [11] indicate large red shifts corresponding to electron densities of $(5-10) \times 10^{23} \mathrm{~cm}^{-3}$ and temperatures of $\sim 300 \mathrm{eV}$. These observations, however, remain a subject of some controversy, particularly due to occurrence of significant high-order satellite emission overlapping the resonance lines and the associated uncertainties introduced by the wavelength markers.

This paper focuses on spectroscopic determination of plasma parameters at the irradiated (front) and unirradiated (rear) surfaces of laser-exploded foils. Taking into account the laterally unconstrained plasma expansion, these measurements are complementary to previously reported experiments on dot targets [8]. The experimental details are presented with emphasis on the high dispersion of the spectrometer used. By using a standard code for density diagnosis [3] and the shift factors computed with a quantum-mechanical impact theory [5], the observed line widths and shifts of the Al Lyman transition are interpreted in terms of the plasma density variation with respect to the distance from the laser-irradiated target. To verify the results obtained, the time-integrated spectroscopic data are compared with synthetic spectra produced by post-processing the $1 \mathrm{D}$ and 2D hydrodynamic simulations. Finally, the conclusions for density diagnostics based on the line shifts in intermediately coupled plasmas are drawn.

\section{Experimental details}

The experiment was carried out on the iodine laser system at the PALS Research Center in Prague [12]. The scheme of the experimental geometry is shown in Fig. 1. A single laser beam delivering up to $220 \mathrm{~J}$ of frequency-tripled radiation $(0.44 \mu \mathrm{m})$ in a pulse length of $0.4 \mathrm{~ns}$ was focused to a diameter of $80 \mu \mathrm{m}$, yielding a maximum intensity of $1.1 \times 10^{16} \mathrm{~W} / \mathrm{cm}^{2}$ on a target. The radiation was incident normally onto self-supported aluminum foils with thickness ranging from 0.8 to $5 \mu \mathrm{m}$. Here, we shall concentrate on an evaluation of the laser interaction with the 5 - $\mu \mathrm{m}$-thick foil characterized by the strongest asymmetry in the plasma parameters distribution at both surfaces of the target. The complementary results measured on thinner foils will be published elsewhere [13].

The principle diagnostics used in this experiment was a vertical-geometry Johann spectrometer (VJS). The VJS disperses the radiation in a direction parallel to the axis of the cylindrically bent crystal, i.e., as a function of the vertical divergence angle $\varphi$. The source and the detector are 


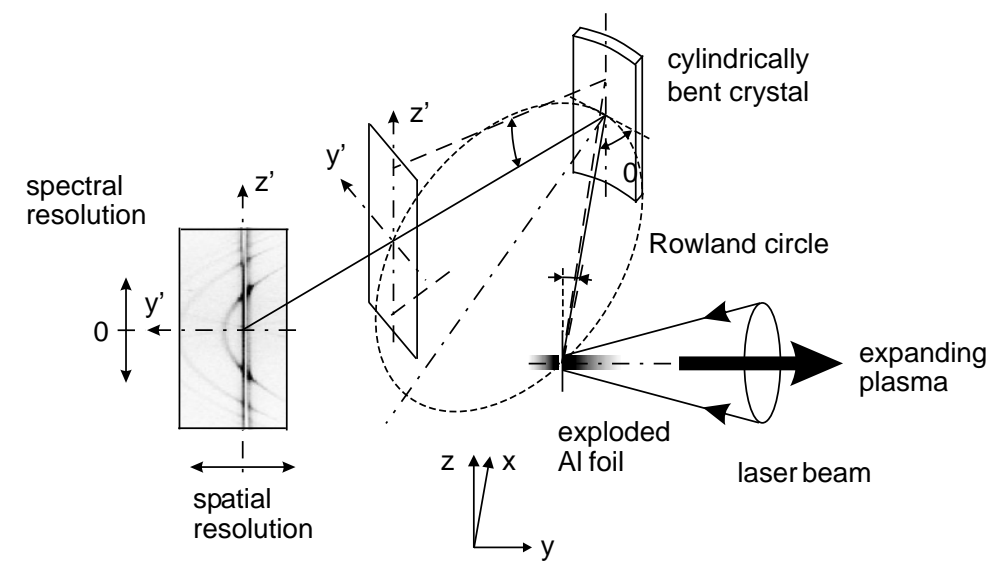

Fig. 1. A schematic diagram of the experiment.

positioned on the Rowland circle defined by the crystal center and the spectra are recorded with 1D spatial resolution of a few microns obtained in a direction perpendicular to the dispersion plane. For sources extended along the $y$-axis, the Bragg angle of the central ray $\theta_{0}$ decreases with the increasing distance of the emitting region from the Rowland circle. This explains the arched character of the spectral lines recorded at the detector (schematically shown in Fig. 1). The instrument provides two identical sets of spectra symmetrically disposed about the central wavelength $\lambda_{0}$ (related via the Bragg equation to the angle $\theta_{0}$ ). The existence of this axis of symmetry facilitates the computational reconstruction of the detected spectra and, what is of primary importance, the problem of accurate measurement of the spectral line positions or their relative changes is reduced to the precise determination of the spacings between corresponding mirror-symmetric spectral features [14].

The VJS was fitted with a crystal of quartz (100) bent to a radius of $77.2 \mathrm{~mm}$ and observed the spectrum at an angle of emission $\psi=1^{\circ}$ to the target surface, the spatial resolution was obtained along the normal to this. The ray-tracing calculations suggest that for the Ly $\delta$ line of hydrogenic aluminum and an angle $\varphi=6^{\circ}$, the VJS provides a spectral resolving power of 3900 , linear dispersion of $175 \mathrm{~mm} / \AA$ (as compared to a value of $16 \mathrm{~mm} / \AA$ provided by a standard flat crystal configuration), and a collection efficiency 2 orders of magnitude higher than a comparable flat-crystal scheme. The spatial resolution was limited to $12 \mu \mathrm{m}$ by the microdensitometer slit width. These characteristics give the VJS distinct advantages over other spectroscopic schemes in this type of measurements.

Due to the extremely high dispersion, the range of the wavelengths $\Delta \lambda$ covered at a given alignment of the VJS is rather limited $(\Delta \lambda / \lambda<0.03)$. At a given angular setting, Al Ly $\delta$ and all resolved higher members of the series (typically up to Ly $\eta$ or $\theta$, i.e., transition $8 p$ or $9 p \rightarrow 1 s$ ) could be observed simultaneously.

The spectra were recorded on X-ray film Kodak Industrex CX, their reconstruction proceeded in three steps. First, the image was digitized using the densitometer with an effective slit size $12 \times 100 \mu \mathrm{m}^{2}$ (spatial resolution $\times$ dispersion direction) and the optical densities were converted to an intensity scale. Second, the spatially resolved spectral lineouts were correlated with the origin of the $y$-axis (perpendicular to the target and measured from the foil surface); the reference transition $\mathrm{Al}$ Ly $\delta$ used to calibrate the spectra was defined at the distance of $144 \mu \mathrm{m}$ from the target. Finally, all 
relevant scans were extracted and recalculated to the linear wavelength scale by using an algorithm described in Ref. [14].

\section{Spectra evaluation}

The signal distribution at the detector shown in the insertion of Fig. 1 is a genuine densitogram of the spectrum emitted from the 5 - $\mu$ m-thick Al foil at the laser intensity of $3 \times 10^{15} \mathrm{~W} / \mathrm{cm}^{2}$. The real size of this scan is $56 \times 1.3 \mathrm{~mm}^{2}$, i.e., the direction of spatial resolution was stretched to fit the picture. The spatially resolved emission of individual spectral lines forms a set of conics; if any irregularity occurs in the emission, this shape is distorted.

The surface plot from the magnified upper part of the insertion from Fig. 1 is shown in Fig. 2. The Lyman series members $\delta, \varepsilon, \zeta$, and $\eta$ can easily be identified. The characteristic features of the spectra, i.e., the variable line ratios, their gradual broadening and merging close to both foil surfaces are obvious. Note that an area of about $30 \mu \mathrm{m}$ behind the foil is obscured by an unablated foil material. We can also easily identify the deformation of spectral conics corresponding to a shift of the spectral lines.

These effects are quantitatively described in Fig. 3. The variable intensities of the reconstructed spectral lineouts represent the actual measured quantities, for clarity the three scans closest to the rear surface, $-36,-48$, and $-60 \mu \mathrm{m}$ were shifted by the values $1.5,0.5$, and $0.1 \mathrm{photons} / \mu \mathrm{m}^{2}$, respectively. At larger distances, higher Lyman series members are well resolved whereas close to the target only the transitions Al Ly $\delta$ and $\varepsilon$ can be distinguished; all other lines merge with the continuum. This phenomenon is distinct enough to be used for rough estimates of the plasma density

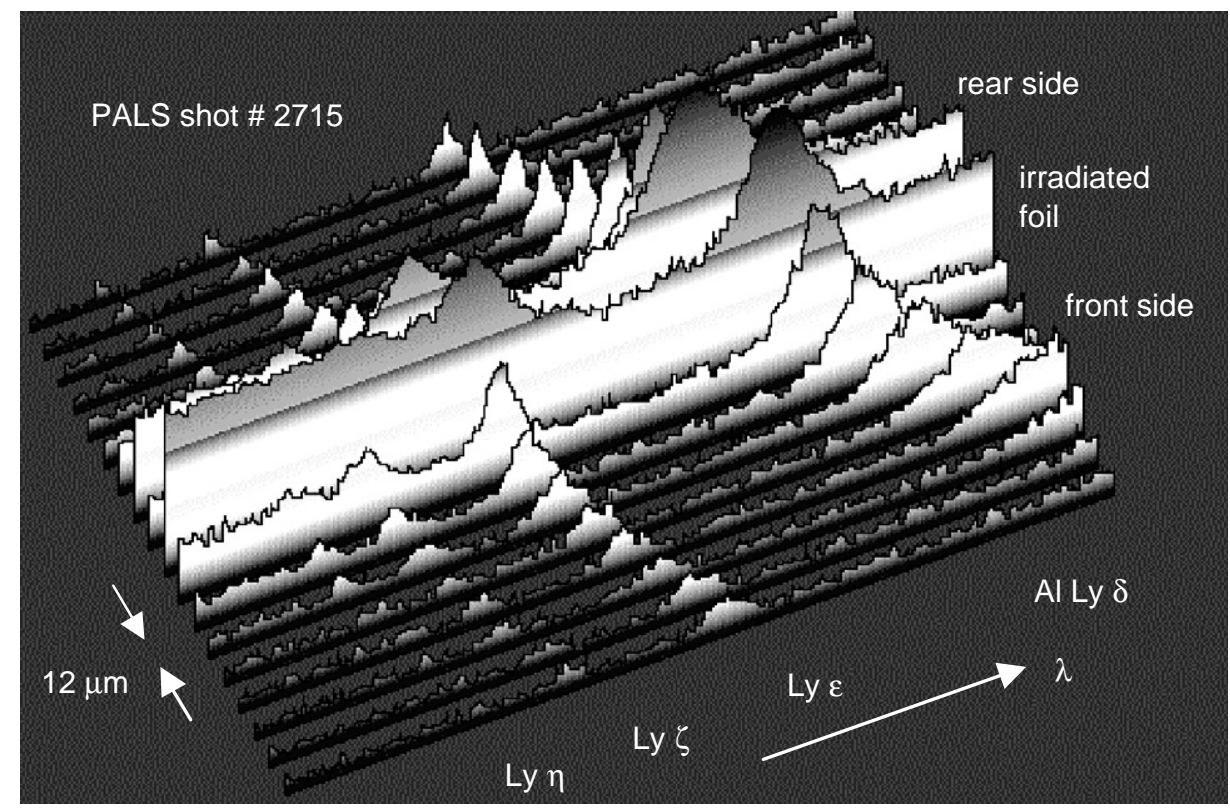

Fig. 2. A part of the symmetric spectra emitted from 5 - $\mu$ m-thick $\mathrm{Al}$ foil irradiated at $3 \times 10^{15} \mathrm{~W} / \mathrm{cm}^{2}$. 

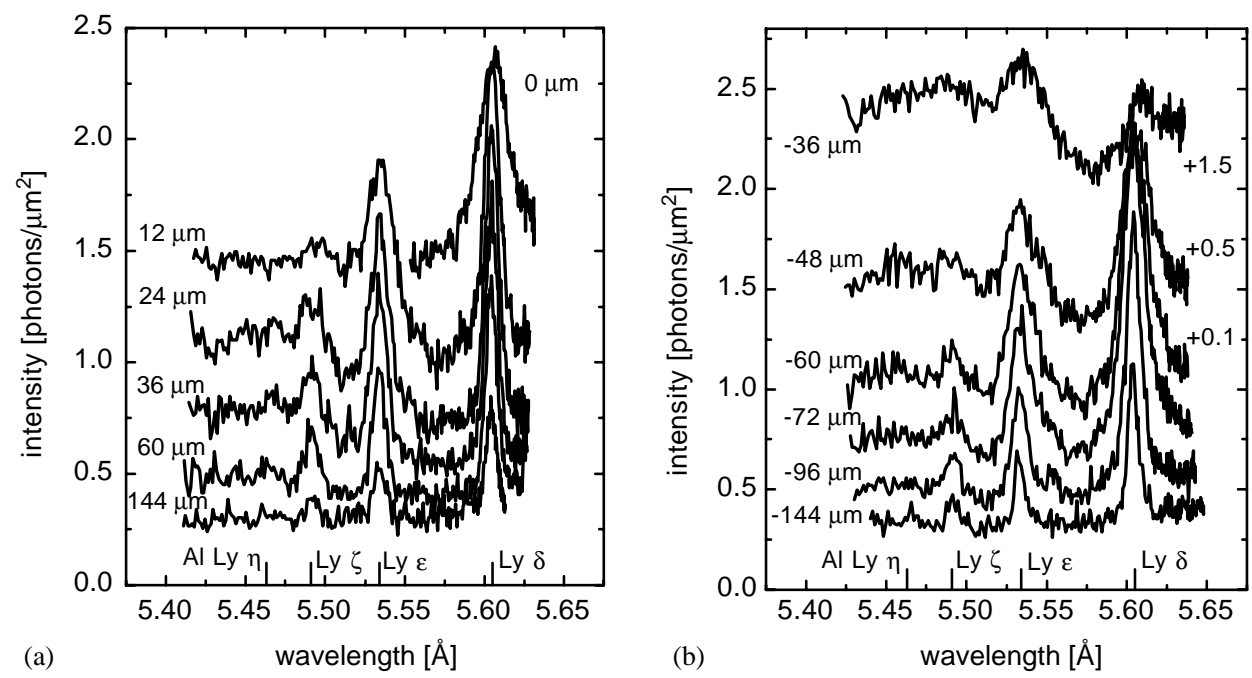

Fig. 3. Spatially resolved spectra observed at the front (a) and rear (b) surface of the laser-exploded foil.

(via Inglis-Teller limit); however, in the following, we shall concentrate on a detailed analysis of the diagnostically important Ly $\delta$ emission profiles.

The spectra background was approximated by a polynomial of the third order and subtracted, the spectral lines were fit with pseudo-Voigt profiles. The starting parameters (line center, amplitude, width and Gaussian-Lorentzian shape factor) were refined using a least-squares procedure. The precision of the line center of gravity determination is estimated to be $\Delta \lambda / \lambda=(0.5-1) \times 10^{-4}$, where $\Delta \lambda$ is the position uncertainty. The main contributions to this error are introduced by the effects of the radiation trapping, as one finds that close to the target the optical depth of the Al Ly $\delta$ approaches 2 , and by uncertainties in the Doppler correction. The intrinsic limit of the VJS for determination of the relative line shifts approaches the value of $\Delta \lambda / \lambda=1 \times 10^{-5}$ [14] that, for a given experimental configuration, corresponds to a $20 \mu \mathrm{m}$ change in spacing of relevant spectral features at the detector. Such shifts can reliably be identified with our high-dispersion spectrometer.

The space-dependent FWHM widths and positions of the Ly $\delta$ transition are shown in Fig. 4. The line widths increase from approximately $6 \mathrm{~m} \AA$ observed at the distance of about $150 \mu \mathrm{m}$ to more than $25 \mathrm{~m} \AA$ close to the irradiated foil surface. This broadening is mostly related to the Stark effect which dominates the line widths at higher densities; for electron density $n_{\mathrm{e}}=10^{20} \mathrm{~cm}^{-3}$ and temperature $T_{\mathrm{e}}=1000 \mathrm{eV}$, theory [3] predicts the FWHM width of $2.9 \mathrm{~m} \AA$ only. The line positions change by approximately $6 \mathrm{~m} \AA$, i.e., about a quarter of the maximum line width recorded. The observed trend in the shifts data supports the idea that there is a density-dependent line shift.

The measured line widths and shifts were converted to electron densities by using the code FLY [3] and the line-shift factors following from the quantum-mechanical impact theory [5]. Both these methods require some knowledge of the electron temperature in the emitting plasma. The needed values were derived from time-integrated spectra taken at similar conditions by a spatially resolving survey spectrometer equipped with a flat ADP (101) crystal [12] and from plasma hydrodynamic simulations described in the next section. 

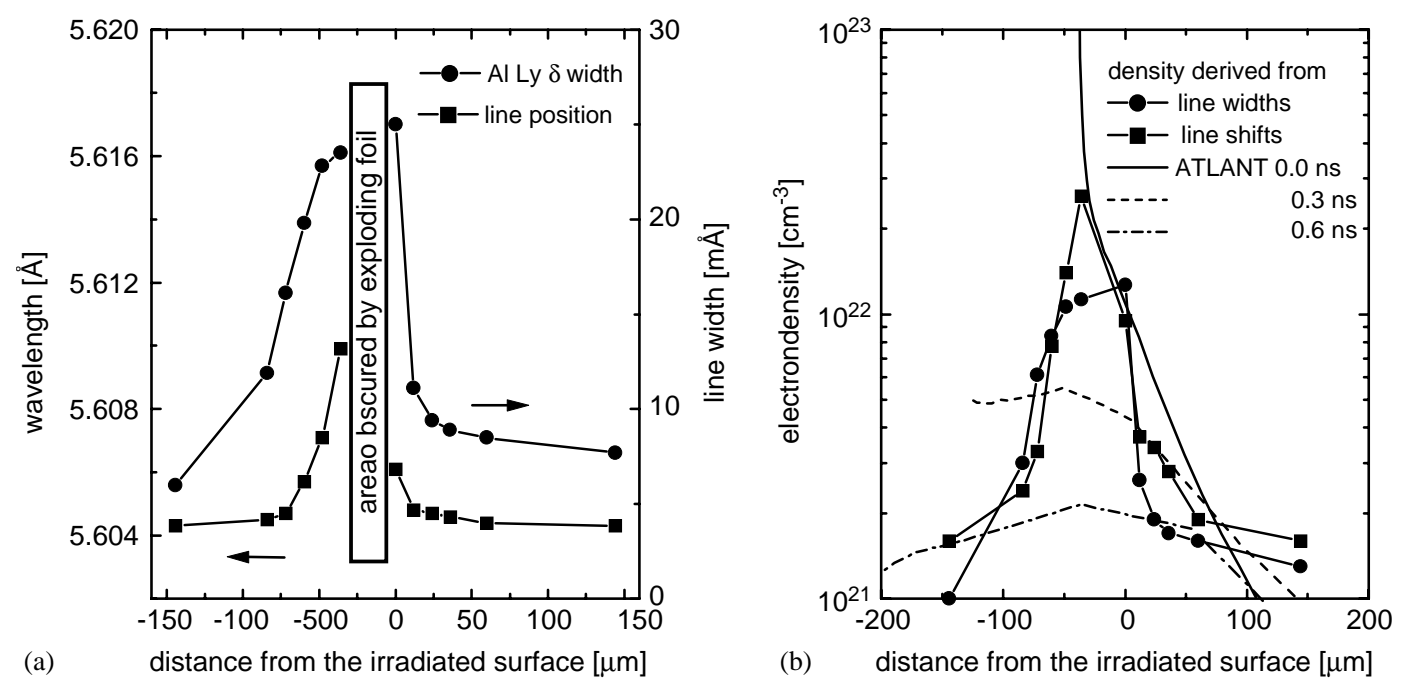

Fig. 4. The space-dependent widths and positions of the Al Ly $\delta$ emission profiles (a) and their recalculation to the electron densities (b). For comparison, the density distributions simulated by the hydrocode ATLANT for three characteristic times related to the laser pulse maximum are shown.

As mentioned already, the Stark-broadened shapes and positions of spectral lines depend strongly on density and relatively weakly on temperature. That is, for a temperature change in the range 500$2000 \mathrm{eV}$ the density derived from the width changes by $\pm 12 \%$ over the density range of interest. The variation of the line positions with respect to temperature can be determined from the theory [5] predicting that for Lyman $n p \rightarrow 1 s$ transitions in hydrogen-like ions the energy shifts are $\Delta h v=$ $-C_{n}\left(n_{\mathrm{e}} / 10^{22} \mathrm{~cm}^{-3}\right)$, where $C_{n}$ is dependent on temperature. For the Al Ly $\delta$, the values of this constant are $C_{n}=1.055,0.903$, and $0.837 \mathrm{eV}$ for $T_{\mathrm{e}}=340,845$, and $1690 \mathrm{eV}$, respectively. Thus, the error introduced typically does not exceed several percent. In the first approximation, we can also neglect the effects of temperature on ground and excited populations, which can modify the radiation transfer and the intensity distribution in the spectra.

The results presented in Fig. $4 \mathrm{~b}$ demonstrate the consistency of the methods based on line widths and shifts for the inference of electron densities in the range of $n_{\mathrm{e}} \geqslant 10^{21}$, i.e., in plasma regions close to the foil surfaces. The validity of these results is supported by the hydrocode simulations, the details of which will be presented in the next section. The density distributions, derived from the simulations for times at laser pulse maximum, +0.3 , and $+0.6 \mathrm{~ns}$ after the maximum, fit the data deduced from the experiment. The partial discrepancies in the values found can be attributed to uncertainties in the theory and to experimental errors; also the detailed treatment of the radiative transport in the laterally expanding plasma might provide slightly different answers. The results obtained, however, confirm that the density diagnosis based on the line shifts is a viable method for these intermediately coupled plasmas.

\section{Numerical simulations}

The rough estimate of the plasma conditions at both surfaces of the foil was obtained from the planar 1D code MEDUSA supplemented by an average-atom model [15]. This code calculates 
the population densities of the ground and excited states of the ions; the latter are proportional to the emission rates of the given transitions. Consequently, the effective plasma characteristics relevant for time-integrated emission at a given spatial coordinate $y$ were calculated by using the emission rate of the line Al Ly $\delta$ as a weighing factor, see Ref. [16] for details. The synthetic spectra were produced by post-processing the average plasma parameters with the atomic physics package FLY [3].

A refined analysis was based on the simulation of the plasma dynamics using the cylindrical version of the 2D hydrocode ATLANT [17], the one-fluid two-temperature Lagrangian code with natural and artificial viscosity, and electron and ion heat conductivity. The heat flux restriction was included via the flux limiter set to $f \approx 0.1$, plasma atomic physics was based on an average atom approximation and the QEOS equation of state [18]. The laser propagation was described by a ray-tracing algorithm including the collisional light absorption in the underdense plasma. The spatial resolution was insufficient to calculate in detail the laser reflection near the critical surface; therefore, a specified portion of laser energy was deposited in the cell where the reflection occurs. The overall absorption efficiency was set to $\eta_{\mathrm{A}} \approx 0.7$. The hydrocode output for the atomic physics post-processor describes the evolution of plasma parameters along the axis of symmetry. The effective radial extent of the plasma was approximated by a minimum of the density and electron temperature characteristic widths, defined as a double distance $r$ at which these parameters decrease to one-half of their on-axis values.

The spatially resolved, time-integrated spectra were simulated with a novel $1 \mathrm{D}$ planar atomic physics post-processor XEPAP [13] developed to model the K-shell emission from transient inhomogeneous plasmas. By using the hydrodynamic parameters produced by ATLANT, the post-processor calculates in detail the local non-stationary populations of charge states, ground and excited levels for Li-, He- and H-like ions. The radiative transport is modeled by using emission-absorption line shapes (both for resonance and selected satellite lines) calculated via the density-matrix theoretical model of general multielectron ion in plasma [19]. The continuum lowering is treated in the StuartPyatt approximation [20], the density-dependent shifts of the spectral lines and also the recombination edge broadening are not included. A specialized module developed for interpretation of dot target experiments has been used to synthesize the spectra emitted parallel to the target surface. The plasma was assumed to be homogenous in the direction tangential to the surface within its variable effective width. The emission was integrated along rays connected with the Lagrangian coordinates, the final time-integrated spectra were then interpolated onto a static spatial grid.

An example of the calculations simulating the explosion of the 5 - $\mu$ m-thick Al foil is shown in Fig. 5. These images broadly correspond to the peak of X-ray emission in the observed spectral region. At the rear side of the irradiated target $(y=0)$, the residual unablated foil can still be distinguished. The plasma conditions on the axis $50 \mu \mathrm{m}$ behind the target surface are $0.02 \mathrm{~g} / \mathrm{cm}^{3}, T_{\mathrm{e}}=$ $770 \mathrm{eV}, n_{\mathrm{e}}=5.2 \times 10^{21} \mathrm{~cm}^{-3}$, and an average ionic charge is 11.8 . The hydrodynamic simulations followed the target dynamics and the hole formation in the foil up to $3 \mathrm{~ns}$ after the laser pulse arrival, which is sufficient for modeling of the K-shell plasma emission.

The observed and synthetic spectra are compared in Fig. 6. At the distance of $50 \mu \mathrm{m}$ from the irradiated foil surface, the overall agreement of the main spectral features and particularly of the Stark-broadened profiles of the Al Ly $\delta$ lines is satisfactory. The measured FWHM value of the Ly $\delta$ profile $(8.7 \pm 0.4 \mathrm{~m} \AA)$ compares well with the value of $9.1 \mathrm{~m} \AA$ simulated by ATLANT and XEPAP. In addition, the spectra produced by MEDUSA and FLY provide somewhat higher FWHM 

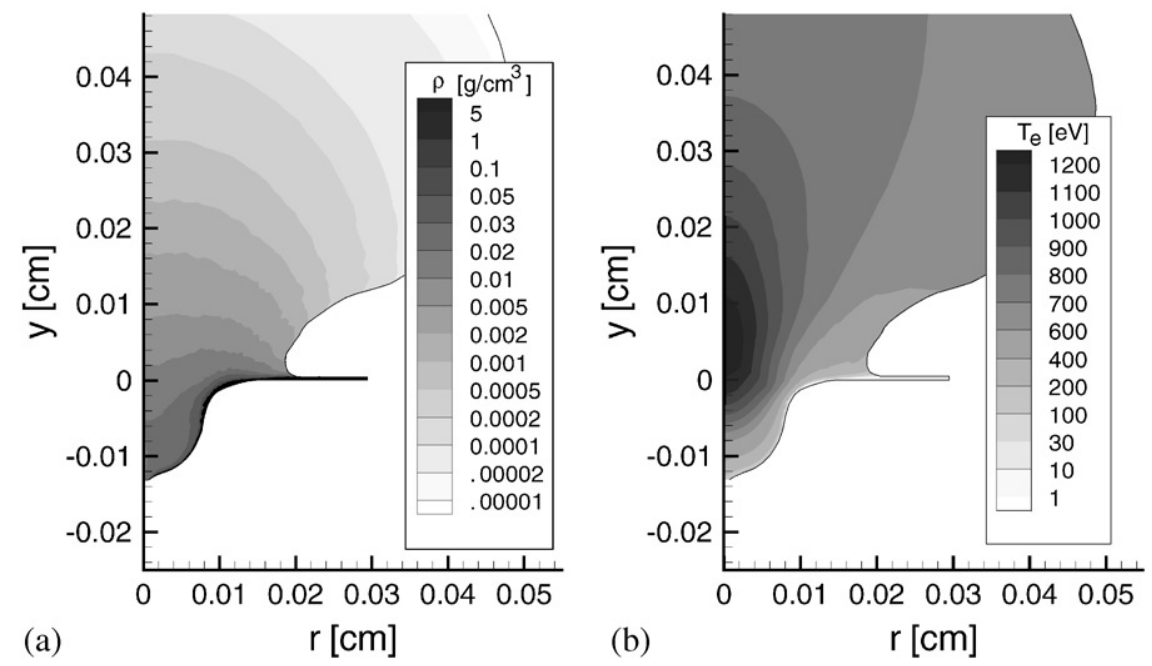

Fig. 5. Plasma density (a) and electron temperature (b) profiles simulated by ATLANT for time $0.3 \mathrm{~ns}$ after the laser maximum. The coordinates $r$ and $y$ correspond to the direction parallel and perpendicular to the foil surface, the laser pulse arrives along the $y$-axis.

values: the Ly $\delta$ line synthesized by post-processing the full set of hydrodynamic data gives the value of $12.4 \mathrm{~m} \AA$ whereas the steady-state synthetic spectrum corresponding to the effective plasma parameters results in the value of $12.1 \mathrm{~m} \AA$. The larger line widths derived from the $1 \mathrm{D}$ hydrodynamic simulation are due to the omission of the energy dissipation in the laterally expanding plasma. By using FLY, the FWHM values of the measured and synthesized Ly $\delta$ profiles infer the effective electron densities $n_{\mathrm{e}}=1.7,2.2,4.2$, and $4.0 \times 10^{21} \mathrm{~cm}^{-3}$, respectively. Comparing the first two values with the electron density derived from the line shift $\left(2.3 \times 10^{21} \mathrm{~cm}^{-3}\right)$, we can conclude that at the front foil surface, both experimental methods provide the data consistent with the $2 \mathrm{D}$ simulation.

At the rear foil surface, the situation is more complicated. At the distance of $-50 \mu \mathrm{m}$, the measured FWHM value of the Ly $\delta$ transition $(22.8 \pm 0.8 \mathrm{~m} \AA)$ corresponds to $n_{\mathrm{e}}=1.1 \times 10^{22} \mathrm{~cm}^{-3}$. This is comparable with the density of $1.4 \times 10^{22} \mathrm{~cm}^{-3}$ derived from the line shift but considerably larger than the values of $7.3 \times 10^{21} \mathrm{~cm}^{-3}$ predicted by ATLANT and XEPAP and $6.2 \times 10^{21} \mathrm{~cm}^{-3}$ produced by a combination of MEDUSA and FLY. The discrepancies in these values cannot be explained by the Doppler effect; according to the calculated ion velocity distribution, the Doppler line broadening should be smaller than $1 \mathrm{~m} \AA$. The explanation may be related to the method used to derive the effective plasma parameters from $2 \mathrm{D}$ hydrodynamic simulations. That is, behind the target, the algorithm chosen leads to underestimated effective densities. The plasma density has its minimum on the axis, thus the average density inside the emitting zone is slightly higher than the axial one; this may be the cause of the differences between the synthetic and observed line widths.

Despite additional limitations arising from the implementation of the hydrocode and the postprocessor-due to limited precision of the energy deposition, neglecting the higher-order dielectronic satellites to the Lyman series members, underestimation of the continuum emission in the synthetic spectra, and simplified calculation of the radiative transfer - the $2 \mathrm{D}$ simulations confirm the observed asymmetry in the plasma parameters at the front and rear surface of the laser-exploded foil. In 

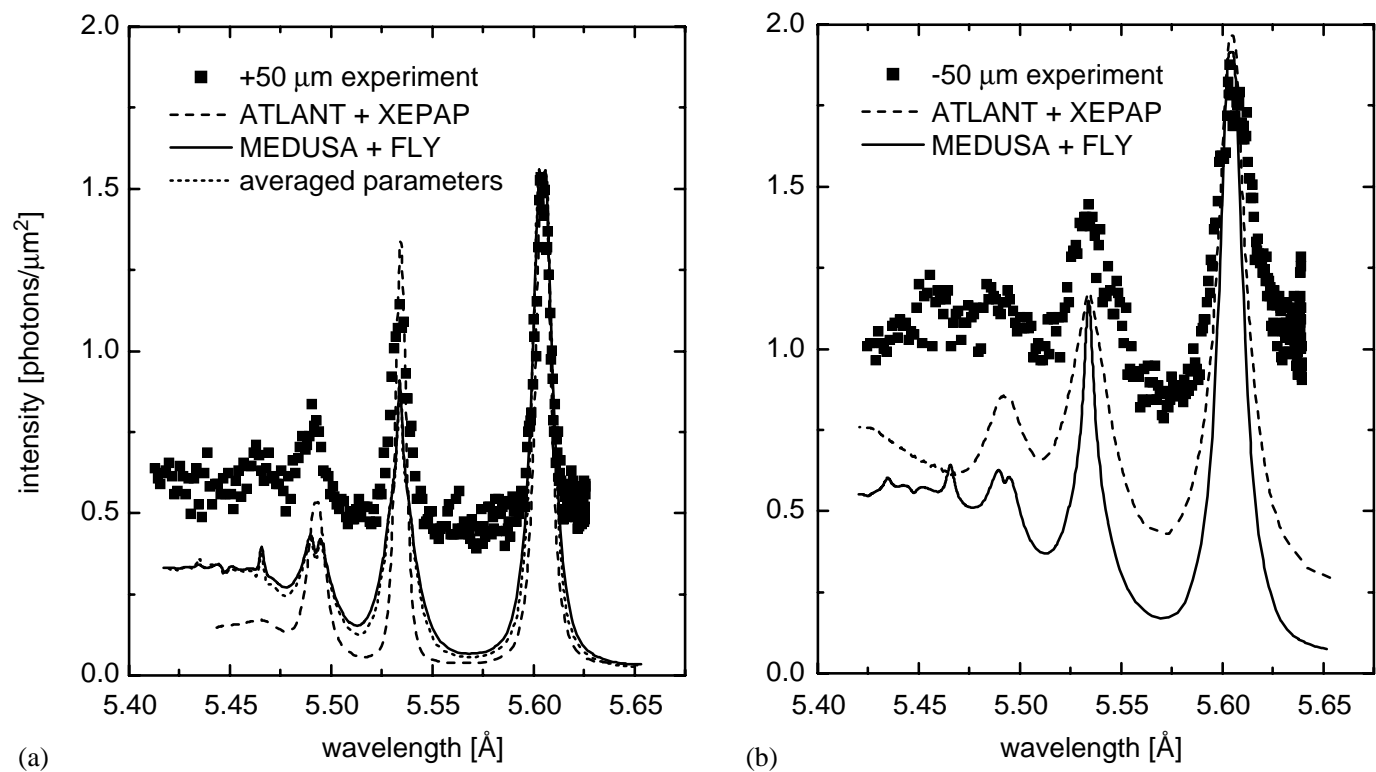

Fig. 6. The comparison of the spectra observed at distances of $50 \mu \mathrm{m}$ from the front (a) and rear (b) target surface with the synthetic spectral profiles. The simulations are based on 2D ATLANT hydrodynamics post-processed by the code XEPAP and 1D MEDUSA modeling combined with the code FLY. For completeness, the steady-state spectrum corresponding to the average plasma parameters is also shown.

particular, the occurrence of the extended dense plasma region behind the target was corroborated. The comparison of the synthesized and experimental data supports the accuracy of the plasma densities derived from the spectral line widths and shifts.

\section{Summary and conclusions}

By using the high-dispersion, spatially resolving VJS, the emission from a laser-irradiated 5- $\mu \mathrm{m}$ thick Al foil was investigated; these measurements are complementary to previously reported experiments with a constrained flow plasma produced on dot targets. The X-ray spectra observed simultaneously at the front and rear surface of the self-supported foil reflect an asymmetry in the distribution of the plasma parameters. The significant features of the spectra emitted from the densest part of the plasma were compared with predictions of post-processed 1D and 2D hydrodynamic simulations. The emission of the Lyman series member $\delta$ from hydrogenic aluminum was analyzed in detail. The widths and relative shifts in the positions of this line were interpreted with respect to the variable density distribution in the expanding plasma. The densities derived from the experiment are consistent with the simulated plasma conditions. The results obtained corroborate the feasibility of the plasma density diagnostics based on the line shifts in the intermediately coupled plasmas. Future work will concentrate on more precise modeling of the laser energy deposition and the spectra synthesis based on the full 2D post-processor with more sophisticated radiative transfer calculations. 


\section{Acknowledgements}

This research was funded by the Czech Grant Agency under Contract No. 202/01/0755, EC Transnational Access to Major Research Infrastructures HPRI-CT-1999-00053 and the CzechGerman Project of Bilateral Cooperation in Science and Technology CZE-00-008. The primary author was supported by a Marie Curie Fellowship of the EC programme Improving the Human Research Potential under Contract No. HPMF-CT-2002-01550.

\section{References}

[1] Griem HR. Principles of plasma spectroscopy. Cambridge: Cambridge University Press, 1997.

[2] Chambers D, Pinto PA, Hawreliak J, Al'Miev IR, Gouveia A, Sondhauss P, Wolfrum E, Wark JS, Glenzer SH, Lee RW, Young PE, Renner O, Marjoribanks RS, Topping S. K-shell spectroscopy of an independently diagnosed uniaxially expanding laser-produced aluminum plasma. Phys Rev E 2002;66:026410-1-16.

[3] Lee RW, Larsen JT. A time dependent model for plasma spectroscopy of K-shell emitters. JQSRT 1996;56:535-56.

[4] Moss C, Calisti A, Stamm R, Talin B, Lee RW, Klein L. Redistribution of resonance radiation in hot dense plasmas. Phys Rev A 1999;60:1005-14.

[5] Nguyen H, Koenig M, Berendjem D, Caby M, Coulaud G. Atomic structure and polarization line shift in dense and hot plasmas. Phys Rev A 1986;33:1279-90.

[6] Junkel GC, Gunderson MA, Hooper CF, Haynes DA. Full Coulomb calculation of Stark broadened spectra from multielectron ions: a focus on the dense plasma line shift. Phys Rev E 2000;62:5584-93.

[7] Skupsky S. X-ray-line shift as a high-density diagnostics for laser-imploded plasmas. Phys Rev A 1980;21:1316-26.

[8] Renner O, Salzmann D, Sondhauss P, Djaoui A, Krousky E, Förster E. Experimental evidence for plasma shifts in Lyman series of aluminum. J Phys B: At Mol Opt Phys 1998;31:1379-90.

[9] Haynes DA, Junkel GC, Gunderson MA, Hooper CF. Plasma induced line shifts and their effects on line merging and population kinetics. In: Mancini RC, Phaneuf RA, editors. Atomic processes in plasmas, vol. CP547. Melville, NY: American Institute of Physics, 2000. p. 227-37.

[10] Woolsey NC, Back CA, Lee RW, Calisti A, Moss C, Stamm R, Talin B, Asfaw A, Klein L. Experimental results on line shifts from dense plasmas. JQSRT 2000;65:573-8.

[11] Saemann A, Eidmann K, Golovkin IE, Mancini RC, Andersson E, Förster E, Witte K. Isochoric heating of solid aluminum by ultrashort laser pulses focused on a tamped target. Phys Rev Lett 1999;82:4843-6.

[12] Jungwirth K, Cejnarova A, Juha L, Kralikova B, Krasa J, Krousky E, Krupickova P, Laska L, Masek K, Mocek T, Pfeifer M, Präg A, Renner O, Rohlena K, Rus B, Skala J, Straka P, Ullschmied J. The Prague Asterix laser system. Phys Plasmas 2001;8:2495-501.

[13] Limpouch J, Renner O, Krousky E, Kralikova B, Skala J, Förster E, Lübcke A, Uschmann I, Iskakov AB, Kocbach L, Loboda PA, Popova VV, Litvinenko IA. X-ray emission diagnostics of the densest part of plasma at laser-exploded foils. In: Guskov SY, editor. Proceedings SPIE 5228, 2003, in print.

[14] Renner O, Missalla T, Sondhauss P, Krousky E, Förster E, Chenais-Popovics C, Rancu O. High-luminosity, high-resolution X-ray spectroscopy of laser-produced plasma by vertical-geometry Johann spectrometer. Rev Sci Instrum 1997;68:2393-403.

[15] Djaoui A, Rose SJ. Calculation of the time-dependent excitation and ionization in laser-produced plasma. J Phys B: At Mol Opt Phys 1992;25:2745-62.

[16] Renner O, Sondhauss P, Peyrusse O, Krousky E, Ramis R, Eidmann K, Förster E. High-resolution measurements of X-ray emission from dense quasi-1D plasma. Laser Particle Beams 1999;17:364-75.

[17] Iskakov AB, Lebo IG, Tishkin VF. 2D numerical simulation of the interaction of high-power laser pulses with plane targets by using ATLANT-C. J Russ Laser Res 2000;21:247-63.

[18] More RM, Warren KH, Young DA, Zimmerman GB. A new quotidian equation of state for hot dense matter. Phys Fluids 1988;31:3059-78.

[19] Loboda PA, Litvinenko IA, Baydin GV, Popova VV, Koltchugin SV. Line shape modeling of multielectron ions in plasmas. Laser Particle Beams 2000;18:275-89.

[20] Stewart JC, Pyatt KD. Astrophys J 1966;144:1203-11. 